ANNALES

POLONICI MATHEMATICI

$88.1(2006)$

\title{
Characterization of global Phragmén-Lindelöf conditions for algebraic varieties by limit varieties only
}

\author{
by Rüdiger W. Braun (Düsseldorf), Reinhold Meise (Düsseldorf) \\ and B. A. TAYLOR (Ann Arbor, MI)
}

\begin{abstract}
For algebraic surfaces, several global Phragmén-Lindelöf conditions are characterized in terms of conditions on their limit varieties. This shows that the hyperbolicity conditions that appeared in earlier geometric characterizations are redundant. The result is applied to the problem of existence of a continuous linear right inverse for constant coefficient partial differential operators in three variables in Beurling classes of ultradifferentiable functions.
\end{abstract}

1. Introduction. The existence of a continuous linear right inverse for a given linear partial differential operator $P(D)$ with constant coefficients on the spaces $C^{\infty}\left(\mathbb{R}^{n}\right) / \mathcal{D}^{\prime}\left(\mathbb{R}^{n}\right)$ or $\mathcal{E}_{\omega}\left(\mathbb{R}^{n}\right) / \mathcal{D}_{\omega}^{\prime}\left(\mathbb{R}^{n}\right)$ was characterized by Meise, Taylor, and Vogt [13] and [14] in various ways, in particular by the condition $\operatorname{PL}(\omega)$ (see Definition 6). It is a condition of Phragmén-Lindelöf type for plurisubharmonic functions on the zero variety $V(P)$ of the symbol $P$. A similar but different condition had been used by Hörmander [10] to characterize the surjectivity of $P(D)$ on the space $\mathcal{A}\left(\mathbb{R}^{n}\right)$ of all real-analytic functions on $\mathbb{R}^{n}$.

Recently, we derived in [5] new necessary conditions for $\operatorname{PL}(\omega)$ which state that the limit varieties $T_{\gamma, d} V(P)$ (see Definition 10) of $V(P)$ with respect to each real simple curve $\gamma$ must satisfy the strong Phragmén-Lindelöf condition (SPL) if $\liminf _{t \rightarrow \infty} \omega(t) / t^{d}=0$. This new condition together with a number of hyperbolicity conditions was then used to give a complete characterization of $\operatorname{PL}(\omega)$ for $n=3$. In the same spirit, a characterization of (SPL) was derived in [7] for algebraic surfaces in $\mathbb{C}^{n}$.

The main result of the present paper is to show that the hyperbolicity conditions mentioned above are redundant, i.e., an algebraic surface $V$ in $\mathbb{C}^{n}$ $\left(\right.$ resp. $\mathbb{C}^{3}$ ) satisfies (SPL) (resp. $\left.\mathrm{PL}(\omega)\right)$ if and only if each limit variety $T_{\gamma, d} V$

2000 Mathematics Subject Classification: Primary 32U05; Secondary 31C10, 32C25.

Key words and phrases: Phragmén-Lindelöf condition, limit variety, continuous linear right inverse, hyperbolicity in conoids, ultradifferentiable function. 
(resp. each limit variety $T_{\gamma, d} V$ for which $\liminf _{t \rightarrow \infty} \omega(t) / t^{d}=0$ ) satisfies (SPL). This new characterization is derived from the ones given in [5] and [7] by the curve selection lemma from real algebraic geometry. It is a simpler geometric description than the old one, since the limit varieties $T_{\gamma, d} V$ which come up are either homogeneous surfaces or surfaces which are products of $\mathbb{C}$ with an algebraic curve in $\mathbb{C}^{n-1}$. In both cases geometric characterizations of (SPL) are known from Braun [1] and [7] (see Proposition 20).

We begin by recalling some definitions and notations that will be applied in what follows.

2. Notation. By $B^{n}(x, \varepsilon)$ we denote the ball of radius $\varepsilon$ around $x$ in $\mathbb{C}^{n}$. If $n=1$, the superscript will be omitted.

3. Definition. Let $\omega:[0, \infty[\rightarrow] 0, \infty[$ be a continuous increasing function with the following properties:

$(\alpha) \omega(2 t)=O(\omega(t))$ as $t \rightarrow \infty$,

$(\beta) \omega(t)=o(t)$ as $t \rightarrow \infty$,

$(\gamma) \log t=O(\omega(t))$ as $t \rightarrow \infty$,

$(\delta) \phi: x \mapsto \omega\left(e^{x}\right)$ is a convex function.

Then for $n \in \mathbb{N}$, the function $\left.\omega: \mathbb{C}^{n} \rightarrow\right] 0, \infty[, \omega(z)=\omega(|z|)$, will be called a weight function.

We say that $\omega$ is a weak weight function if $\omega$ is either a weight function or identically 0 .

4. Definition. Let $\omega$ be a weight function that satisfies, instead of $(\beta)$, the stronger condition

$$
(\beta)^{\prime} \int_{0}^{\infty} \frac{\omega(t)}{1+t^{2}} d t<\infty .
$$

Then the space of all ultradifferentiable functions of Beurling type on $\mathbb{R}^{n}$ is defined as $\mathcal{E}_{\omega}\left(\mathbb{R}^{n}\right):=\left\{f \in C^{\infty}\left(\mathbb{R}^{n}\right):\right.$ for each $K \subset \mathbb{R}^{n}$ compact and each $m \in \mathbb{N}$,

$$
\left.\sup _{\alpha \in \mathbb{N}_{0}^{n}} \sup _{x \in K}\left|f^{(\alpha)}(x)\right| \exp \left(-m \phi^{*}(|\alpha| / m)\right)<\infty\right\},
$$

where $\phi^{*}(y):=\sup _{x>0}(x y-\phi(x))$ for $y>0$.

For a systematic study of these spaces and the corresponding spaces of ultradistributions we refer to [2].

5. Notation. For $P \in \mathbb{C}\left[z_{1}, \ldots, z_{n}\right], P(z)=\sum_{|\alpha| \leq m} a_{\alpha} z^{\alpha}$, define the partial differential operator $P(D):=\sum_{|\alpha| \leq m} a_{\alpha} i^{-i|\alpha|} \partial^{\alpha}$.

We denote by $V(P):=\left\{z \in \mathbb{C}^{n}: P(z)=0\right\}$ the variety of the symbol of $P(D)$. 
Meise, Taylor, and Vogt [14] were able to decide, for any given polynomial $P$, whether the operator $P(D): \mathcal{E}_{\omega}\left(\mathbb{R}^{n}\right) \rightarrow \mathcal{E}_{\omega}\left(\mathbb{R}^{n}\right)$ admits a continuous linear right inverse. To state their theorem, the Phragmén-Lindelöf condition $\mathrm{PL}(\omega)$ is needed.

6. Definition. Let $V$ be an algebraic variety in $\mathbb{C}^{n}$ and let $\omega$ be a weak weight function.

(a) A function $u: V \rightarrow[-\infty, \infty$ [ is plurisubharmonic if it is locally bounded above, plurisubharmonic in the usual sense at all regular points of $V$ (i.e., via coordinates), and satisfies

$$
u(z)=\limsup _{\substack{\zeta \rightarrow z \\ \zeta \in V \text { regular }}} u(\zeta)
$$

at the singular points of $V$. By $\operatorname{PSH}(V)$ we denote the set of all plurisubharmonic functions on $V$.

(b) $V$ satisfies the Phragmén-Lindelöf condition $\mathrm{PL}(\omega)$ if there exists $A \geq 1$ such that for each $\varrho>0$ there is $B>0$ such that each $u \in \operatorname{PSH}(V)$ which satisfies $(\alpha)$ and $(\beta)$ also satisfies $(\gamma)$, where

$(\alpha) u(z) \leq|z|+o(|z|), z \in V$,

( $\beta) u(z) \leq \varrho|\operatorname{Im} z|, z \in V$,

$(\gamma) u(z) \leq A|\operatorname{Im} z|+B \omega(z), z \in V$.

(c) The Phragmén-Lindelöf condition PL(0) is denoted by (SPL).

7. Remark. To see that (SPL) as defined in Definition 6 coincides with the original definition, given by Meise and Taylor in [12, Definition 3.1(c)], note that our definition is clearly weaker than the one by Meise and Taylor, but stronger than their property $\widetilde{\mathrm{SPL}}(A, 0)$ for a suitable constant $A$. Hence they are equivalent by [12, Lemma 3.2].

8. Theorem (Meise, Taylor, and Vogt [14, Theorem 5.5]). Assume that the weight function $\omega$ satisfies $(\beta)^{\prime}$ from Definition 4 . Then $P(D): \mathcal{E}_{\omega}\left(\mathbb{R}^{n}\right) \rightarrow$ $\mathcal{E}_{\omega}\left(\mathbb{R}^{n}\right)$ admits a continuous linear right inverse if and only if $V(P)$ satisfies $\operatorname{PL}(\omega)$.

It has been shown in [3], [5], and [7] that an important tool for the investigation of Phragmén-Lindelöf conditions are limit varieties. The corresponding definitions are given next.

9. Definition. A real simple curve $\gamma$ is a map $\gamma:] \alpha, \infty\left[\rightarrow \mathbb{R}^{n}\right.$ which for some $\alpha>0$ and some $q \in \mathbb{N}$ admits a convergent expansion

$$
\gamma(t)=\sum_{j=-\infty}^{q} a_{j} t^{j / q}, \quad \text { with }\left|a_{q}\right|=1 .
$$

The vector $a_{q}$ is the limit vector of $\gamma$ at infinity. 
10. Definition. Let $V \subset \mathbb{C}^{n}$ be an algebraic variety, let $\left.\gamma:\right] \alpha, \infty\left[\rightarrow \mathbb{R}^{n}\right.$ be a real simple curve, and let $d \leq 1$.

(a) For $t \in] \alpha, \infty[$ we define

$$
V_{t}:=\left\{w \in \mathbb{C}^{n}: \gamma(t)+w t^{d} \in V\right\}
$$

and we define the limit variety $T_{\gamma, d} V$ of $V$ of order $d$ along $\gamma$ as $T_{\gamma, d} V=\left\{\zeta \in \mathbb{C}^{n}: \zeta=\lim _{j \rightarrow \infty} z_{j}\right.$ where $z_{j} \in V_{t_{j}}$ for $j \in \mathbb{N}$ and

$\left(t_{j}\right)_{j \in \mathbb{N}}$ is a sequence in $] \alpha, \infty[$ which tends to $\infty\}$.

(b) The tangent cone $T_{p} V$ at a point $p \in \mathbb{C}^{n}$ consists of all limits $v=\lim _{j \rightarrow \infty} a_{j}\left(p_{j}-p\right)$ where $p_{j} \in V$ and $a_{j} \in \mathbb{C}$ for $j \in \mathbb{N}$ and $\lim _{j \rightarrow \infty} p_{j}=p$.

(c) The cone of limiting directions is defined as

$$
V_{h}:=\left\{r \lim _{j \rightarrow \infty} \frac{z_{j}}{\left|z_{j}\right|}: r \geq 0, z_{j} \in V \text { with }\left|z_{j}\right| \rightarrow \infty\right\} .
$$

11. Remark. We collect some basic properties of limit varieties. To do so, we fix $d \leq 1$ and a real simple curve $\gamma$.

(a) By [6, Theorem 1], limit varieties are algebraic.

(b) If $\xi$ is the limit vector of $\gamma$ at infinity, then $T_{\gamma, 1} V=V_{h}-\xi$. If $d<1$, then $T_{\gamma, d} V$ is invariant under arbitrary translations in the direction of $\xi$. This was shown in [6, Proposition 4].

(c) It was shown in [6, Proposition 5] that there are numbers $1=: d_{1}>$ $d_{2}>\cdots>d_{p}$ with $p \geq 1$ such that the map $d \mapsto T_{\gamma, d} V$ is constant on the intervals $] d_{j+1}, d_{j}[, j=1, \ldots, p-1$, as well as on the interval ]$-\infty, d_{p}[$.

(d) If $d_{j}>d>d_{j+1}$ for some $j<p$, then

$$
T_{0}\left(T_{\gamma, d_{j}} V\right)=T_{\gamma, d} V=\left(T_{\gamma, d_{j+1}}\right)_{h} .
$$

If $d<d_{p}$, then

$$
T_{0}\left(T_{\gamma, d_{p}} V\right)=T_{\gamma, d} V
$$

Both statements are shown in [8, Corollary 4.15]. In particular, $T_{\gamma, d} V$ is homogeneous unless $d$ is one of the $d_{j}$.

12. Definition. Assume that $\left\{d_{1}, \ldots, d_{p}\right\}$ is the minimal set such that Remark 11(c) holds. Then the numbers $1=d_{1}>d_{2}>\cdots>d_{p}$ are called critical values for $\gamma$ and $V$.

13. Definition. Let $V$ be an algebraic variety in $\mathbb{C}^{n}$ of pure dimension $k \geq 1$. A projection $\pi: \mathbb{C}^{n} \rightarrow \mathbb{C}^{n}$ is called noncharacteristic for $V$ at $\zeta \in V$ if its rank is $k$, its image and its kernel are spanned by real vectors, and $T_{\zeta} V \cap \operatorname{ker} \pi=\{0\}$. It is called noncharacteristic for $V$ at infinity if its rank is $k$, its image and its kernel are spanned by real vectors, and $V_{h} \cap \operatorname{ker} \pi=\{0\}$. 
14. Definition. Let $V$ be an algebraic surface in $\mathbb{C}^{n}$ and let $\gamma$ be a real simple curve. If $d<1$ is a critical value for $\gamma$, then a real, singular point $\zeta$ of $T_{\gamma, d} V$ is called a simple singularity if the curve $\gamma_{\zeta}: t \mapsto \gamma(t)+\zeta t^{d}$ admits no critical value strictly smaller than $d$. If $d=1$, then a real, singular point $\zeta$ of $V_{h}$ is called a simple singularity if the curve $\gamma_{\zeta}: t \mapsto t \zeta$ admits no critical value strictly smaller than 1 . If $d \leq 1$ is a critical value for $\gamma$ and if a real singularity $\zeta$ of $T_{\gamma, d} V$ is not a simple singularity, then $\Delta(\gamma, d, \zeta)$ denotes the largest critical value for $\gamma_{\zeta}$ which is strictly smaller than $d$.

15. Definition. Let $\gamma:\left[\alpha, \infty\left[\rightarrow \mathbb{R}^{n}\right.\right.$ be a real simple curve, let $d \leq 1$, let $U \subset \mathbb{C}^{n}$, and let $R \geq \alpha$. Then the set

$$
\Gamma(\gamma, d, U, R):=\bigcup_{t>R}\left(\gamma(t)+t^{d} U\right)
$$

is called a conoid with core $\gamma$, opening exponent $d$, and profile $U$, with tip truncated at $R$.

16. Definition. Let $V$ be an algebraic variety in $\mathbb{C}^{n}$ of pure dimension $k \geq 1$, let $\gamma:\left[\alpha, \infty\left[\rightarrow \mathbb{R}^{n}\right.\right.$ be a real simple curve, let $d \leq 1$, let $\zeta \in T_{\gamma, d} V \cap \mathbb{R}^{n}$, and let $\omega$ be a weak weight function.

(a) We say that $V$ is $(\omega, \gamma, d)$-hyperbolic at $\zeta$ with respect to a projection $\pi: \mathbb{C}^{n} \rightarrow \mathbb{C}^{n}$ which is noncharacteristic for $T_{\gamma, d} V$ at $\zeta$ if there exist a neighborhood $U$ of $\zeta, r>\alpha$, and $C>0$ such that $z \in V \cap \Gamma(\gamma, d, U, r)$ satisfies $|\operatorname{Im} z| \leq C \omega(z)$ whenever $\pi(z)$ is real.

(b) Let $\delta<d$. We say that $V$ is $(\omega, \gamma, d, \delta)$-hyperbolic at $\zeta$ if there exist $R>1$, a neighborhood $U$ of $\zeta$, a bounded neighborhood $G$ of $\zeta$, and $C>0$ such that $|\operatorname{Im} z| \leq C \omega(z)$ whenever $z \in \Gamma(\gamma, d, U, R)$ satisfies $\pi(z) \in \mathbb{R}^{n}$ and $\pi(z) \notin \Gamma(\pi \circ \gamma, \delta, \pi(G), 0)$.

17. Definition. Let $V$ be an algebraic surface in $\mathbb{C}^{n}$ and let $\omega$ be a weak weight. We say that $V$ is $\omega$-hyperbolic in conoids if for each real simple curve $\gamma$ and each $d \leq 1$ which satisfies $\liminf _{t \rightarrow \infty} \omega(t) / t^{d}=0$ the following conditions are satisfied:

(a) $T_{\gamma, d} V$ satisfies condition $\mathrm{PL}_{\text {loc }}(\xi)$ (see Definition 19 below) for each $\xi \in T_{\gamma, d} V \cap \mathbb{R}^{n}$.

(b) $V$ is $(\omega, \gamma, d)$-hyperbolic at $\xi$ whenever one of the following conditions is satisfied:

(i) $\xi \in\left(T_{\gamma, d} V\right)_{\text {reg }} \cap \mathbb{R}^{n}$,

(ii) $\xi \in \mathbb{R}^{n}$ is a simple singularity of $T_{\gamma, d} V$, and $d$ is a critical value for $\gamma$ and $V$.

(c) If $d$ is a critical value for $\gamma$ and $V$, then for each $\xi \in\left(T_{\gamma, d} V\right)_{\operatorname{sing}} \cap \mathbb{R}^{n}$ which is not a simple singularity of $T_{\gamma, d} V$, the variety $V$ is $(\omega, \gamma, d, \delta)$ hyperbolic at $\xi$ for $\delta=\Delta(\gamma, d, \xi)$. 
If $\omega$ is a weight function, then the significance of the notion of " $\omega$ hyperbolicity in conoids" is explained by [5, Theorem 4.7]. It implies the following result.

18. TheOREM. Let $V$ be an algebraic surface in $\mathbb{C}^{3}$ and let $\omega$ be a weight function. Then $V$ satisfies $\mathrm{PL}(\omega)$ if and only if $V$ is $\omega$-hyperbolic in conoids.

We want to show that for algebraic surfaces in $\mathbb{C}^{n}$ conditions (b) and (c) of Definition 17 are redundant once condition (a) is replaced by the stronger condition that $T_{\gamma, d} V$ satisfies (SPL). Condition (SPL) was characterized for curves and surfaces in $\mathbb{C}^{n}$ in [7]. To state these characterizations we need the local Phragmén-Lindelöf condition $\mathrm{PL}_{\mathrm{loc}}(\xi)$, which was introduced by Hörmander [10]. For a geometric characterization of $\mathrm{PL}_{\text {loc }}(\xi)$ for analytic curves in $\mathbb{C}^{n}$ and analytic surfaces in $\mathbb{C}^{3}$ we refer to [3, Proposition 3.16 and Theorem 5.5]. Next we recall the definition of $\mathrm{PL}_{\text {loc }}(\xi)$ and the characterization of (SPL) for algebraic curves in $\mathbb{C}^{n}$.

19. Definition. For $\xi \in \mathbb{R}^{n}$ and $r_{0}>0$ let $V$ be an analytic variety in $B\left(\xi, r_{0}\right)$ which contains $\xi$. We say that $V$ satisfies condition $\mathrm{PL}_{\text {loc }}(\xi)$ if there exist positive numbers $A$ and $r_{0} \geq r_{1} \geq r_{2}$ such that each $u \in$ $\operatorname{PSH}\left(V \cap B\left(\xi, r_{1}\right)\right)$ satisfying $(\alpha)$ and $(\beta)$ also satisfies $(\gamma)$, where

$(\alpha) u(z) \leq 1$ for $z \in V \cap B\left(\xi, r_{1}\right)$,

( $\beta) u(z) \leq 0$ for $z \in V \cap \mathbb{R}^{n} \cap B\left(\xi, r_{1}\right)$,

$(\gamma) u(z) \leq A|\operatorname{Im} z|$ for $z \in V \cap B\left(\xi, r_{2}\right)$.

20. Proposition ([7, Theorem 3.10]). For each algebraic curve $V$ in $\mathbb{C}^{n}$ the following conditions are equivalent:

(a) $V$ satisfies (SPL),

(b) $V$ satisfies $\mathrm{PL}_{\text {loc }}(\xi)$ for each $\xi \in V \cap \mathbb{R}^{n}$, the cone $V_{h}$ of limiting directions satisfies (SPL), and for each projection $\pi: \mathbb{C}^{n} \rightarrow \mathbb{C}^{n}$ which is noncharacteristic for $V_{h}$ at infinity with $\pi\left(\mathbb{C}^{n}\right)=\mathbb{C} \zeta$ for some $\zeta \in \mathbb{R}^{n}$ there exists $K \geq 1$ such that $\pi^{-1}(t \zeta) \cap V \subset \mathbb{R}^{n}$ for each $t \in \mathbb{R}$ with $|t| \geq K$.

Example 5.2 in [7] shows that in general the introduction of $K \geq 1$ in the previous statement cannot be avoided. However, we are going to show in Lemma 21 and Proposition 22 that under suitable additional hypotheses on the geometry of $V$ the statement of Proposition 20 holds with $K=0$. These two results are crucial for the main result of the present paper.

21. Lemma. Let $V \subset \mathbb{C}^{n-1}$ be an algebraic curve of degree $m$ such that $V_{h}=\left\{z \in \mathbb{C}^{n-1}: z_{2}=\cdots=z_{n-1}=0\right\}$ and such that (SPL) is satisfied for $V$. Then there are $y_{1}, \ldots, y_{m} \in \mathbb{R}^{n-2}$ such that

$$
V=\mathbb{C} \times\left\{y_{1}, \ldots, y_{m}\right\} .
$$


Proof. Let $W$ be a component of $V$ that is locally irreducible at infinity. By the existence theorem for Puiseux series expansions (see, e.g., Chirka $[9,6.1])$, there are $q \in \mathbb{N}$ and holomorphic functions

$$
f_{k}(t)=\sum_{j=-\infty}^{q-1} a_{k, j} t^{j}, \quad k=2, \ldots, n-1,
$$

converging for large complex $t$, such that each point of $W \backslash B(0, R)$ for sufficiently large $R$ has the form

$$
w(t)=\left(t^{q}, f_{2}(t), \ldots, f_{n-1}(t)\right) .
$$

We may assume that $q$ is chosen to be minimal with this property. It is clear from Proposition 20 that all $a_{k, j}$ are real. We claim that $q=1$. Assume for contradiction that $q \geq 2$, and let $\lambda$ be a primitive $q$ th root of -1 . Then, for all sufficiently large $t>0$ the point $w(\lambda t)$ is in $W$. By the minimality condition on $q$ there are arbitrarily large positive values of $t$ for which this point is not real although the first component of $w(\lambda t)$ is real. Since this contradicts Proposition 20, the assumption is false and we have shown $q=1$. Note that this implies that all $f_{k}$ are bounded.

Consider now the canonical defining function of $V$. To define it, set $z=\left(z_{1}, z^{\prime}\right)$ with $z^{\prime}=\left(z_{2}, \ldots, z_{n-1}\right)$. Since $V$ is an $m$-sheeted cover of $\mathbb{C} \times$ $\{(0, \ldots, 0)\}$, there are, for each $z_{1} \in \mathbb{C}$, exactly $m$ vectors $\alpha_{1}\left(z_{1}\right), \ldots, \alpha_{m}\left(z_{1}\right)$ $\in \mathbb{C}^{n-2}$ such that $\left(z_{1}, \alpha_{j}\left(z_{1}\right)\right) \in V$, provided multiplicities are taken into account. Now define

$$
\Phi(z, \xi)=\prod_{j=1}^{m}\left\langle\alpha_{j}\left(z_{1}\right)-z^{\prime}, \xi\right\rangle, \quad z \in \mathbb{C}^{n-1}, \xi \in \mathbb{C}^{n-2} .
$$

This function, which is analytic in $z$ and $\xi$, is the canonical defining function of $V$ with respect to the projection $z \mapsto\left(z_{1}, 0, \ldots, 0\right)$ (for details see Chirka $[9, \S 4.2])$.

Fix now $z^{\prime}, \xi \in \mathbb{C}^{n-2}$. The argument in the first part of this proof shows that the function $z_{1} \mapsto \Phi\left(\left(z_{1}, z^{\prime}\right), \xi\right)$ is bounded for large $z_{1}$ and hence everywhere. By Liouville's theorem it is constant. Hence there are $y_{1}, \ldots, y_{m} \in \mathbb{C}^{n-2}$ such that

$$
\Phi(z, \xi)=\prod_{j=1}^{m}\left\langle y_{j}-z^{\prime}, \xi\right\rangle, \quad z \in \mathbb{C}^{n-1}, \xi \in \mathbb{C}^{n-2} .
$$

Since $z \in V$ if and only if $\Phi(z, \xi)=0$ for all $\xi$, we have shown (1). That the $y_{j}$ are actually real now follows again from Proposition 20.

20. Proposition. Let $V$ be an algebraic surface in $\mathbb{C}^{n}$, let $\gamma$ be a real simple curve, let $d \leq 1$, and assume that 0 is a regular point of $T_{\gamma, d} V$. Fix $\delta<d$ and assume that $T_{\gamma, \varrho} V$ satisfies (SPL) for all $\varrho$ with $d \geq \varrho \geq \delta$. Then 
$T_{\gamma, \delta} V$ is either empty or the union of a finite number of planes which are parallel to $T_{0}\left(T_{\gamma, d} V\right)$ and which have 2-dimensional intersection with $\mathbb{R}^{n}$.

Proof. We only treat the case that $T_{\gamma, \delta} V$ is not empty.

Let $1=d_{1}>d_{2}>\cdots>d_{p}$ be the critical values for $V$ and $\gamma$. Assume first $d \leq d_{p}$ and fix $\delta<d$. By Remark 11(d), $T_{\gamma, \delta} V=T_{0}\left(T_{\gamma, d} V\right)$, which is a plane since 0 is a regular point of $T_{\gamma, d} V$.

To complete the proof by induction, we assume that the assertion holds for all $d \leq d_{j+1}$ and $j+1 \geq p$. Then we fix $d$ with $d_{j+1}<d \leq d_{j}$ and $\delta<d$. If $\delta>d_{j+1}$, then it follows from Remark 11(d) that $T_{\gamma, \delta} V=T_{0}\left(T_{\gamma, d_{j}} V\right)=$ $T_{0}\left(T_{\gamma, d} V\right)$ is a plane which satisfies (SPL). Hence it intersects $\mathbb{R}^{n}$ in a real plane.

Next note that for $\delta=d_{j+1}$, again by Remark 11(d), we also have

$$
\left(T_{\gamma, d_{j+1}} V\right)_{h}=\left(T_{\gamma, \delta} V\right)_{h}=T_{0}\left(T_{\gamma, d} V\right) .
$$

Hence Lemma 21 implies that $T_{\gamma, d_{j+1}} V$ is a finite union of planes which are parallel to $T_{0}\left(T_{\gamma, d} V\right)$, each of which intersects $\mathbb{R}^{n}$ in a real plane. Hence the assertion of the proposition holds for $\delta \in\left[d_{j+1}, d[\right.$.

If $\delta<d_{j+1}$, then $T_{0}\left(T_{\gamma, d_{j+1}} V\right) \neq \emptyset$ since $T_{\gamma, \delta} V \neq \emptyset$. As we have just shown, $T_{0}\left(T_{\gamma, d_{j+1}} V\right)=T_{0}\left(T_{\gamma, d} V\right)$. Therefore, we can apply the induction hypothesis with $d=d_{j+1}$ to complete the proof of the induction step.

The next result is the main tool of the present paper. It states that (b) and (c) of Definition 17 are redundant once in (a) condition $\mathrm{PL}_{\mathrm{loc}}(\xi)$ is replaced by (SPL).

23. Proposition. Let $V$ be an algebraic surface in $\mathbb{C}^{n}$ and let $\omega$ be a weak weight function. Then $V$ is $\omega$-hyperbolic in conoids if and only if $T_{\gamma, d} V$ satisfies (SPL) for all real simple curves $\gamma$ and all $d \leq 1$ with $\liminf _{t \rightarrow \infty} \omega(t) / t^{d}=0$.

Proof. Necessity of the condition is known from [5, Remark 3.29] if $\omega$ is a weight function, and from [7, Corollary 4.4] if $\omega \equiv 0$.

We prove sufficiency by contradiction. To do so, assume that $V$ is not $\omega$-hyperbolic in conoids. Then one of the conditions (a), (b), or (c) in Definition 17 is violated. This cannot be (a), since (SPL) for a variety $W$ implies $\mathrm{PL}_{\text {loc }}(\xi)$ for all $\xi \in W \cap \mathbb{R}^{n}$ by Meise and Taylor [12, Proposition 4.4].

Let us begin with the case that condition (c) is violated. Then there exist a critical value $d \leq 1$ satisfying $\liminf _{t \rightarrow \infty} \omega(t) / t^{d}=0$ and $\xi \in\left(T_{\gamma, d} V\right)_{\text {sing }}$ $\cap \mathbb{R}^{n}$ which is not a simple singularity of $T_{\gamma, d} V$ such that $V$ is not $(\omega, \gamma, d, \delta)$ hyperbolic at $\xi$ for $\delta=\Delta(\gamma, d, \xi)$. Without loss of generality we may assume $\xi=0$. Furthermore, we may assume that $(0, \ldots, 0,1)$ is the limit vector of $\gamma$ at infinity and that $\gamma_{n}(t)=t$ for all $t$, where $\gamma_{n}(t)$ denotes the last coordinate of $\gamma(t)$. We define a projection $\pi$ by $\pi\left(z_{1}, \ldots, z_{n}\right)=\left(0, z_{2}, \ldots, z_{n}\right)$ and assume that coordinates are so chosen that $\pi$ is noncharacteristic for 
$T_{\gamma, d} V$ at the origin, i.e., there are $r, C_{1}>0$ such that

$$
\left|\left(z_{1}, \ldots, z_{n}\right)\right| \leq C_{1}\left|\left(0, z_{2}, \ldots, z_{n}\right)\right|
$$

whenever $\left(z_{1}, \ldots, z_{n}\right) \in T_{\gamma, d} V$ with $\left|\left(z_{1}, \ldots, z_{n}\right)\right|<r$. Note that by Remark 11 the projection $\pi$ is also noncharacteristic for $T_{\gamma, \delta} V$ at infinity.

The proof starts with the same construction as at the beginning of the proof of [4, Proposition 17]. For $d<1$, we choose coordinates in such a way that there are $\varepsilon_{1}, \varepsilon_{2}, K_{1}, K_{2}>0$ such that

(i) If

$$
\left(z_{1}, \ldots, z_{n}\right) \in T_{\gamma, d} V \cap\left(B\left(0, \varepsilon_{1}\right) \times B^{n-2}\left(0,2 \varepsilon_{2}\right) \times \mathbb{C}\right)
$$

satisfies $\left(z_{2}, \ldots, z_{n}\right) \in \mathbb{R}^{n-1}$, then also $z_{1}$ is real. Such numbers $\varepsilon_{1}, \varepsilon_{2}$ exist by [3, Proposition 3.16], since $T_{\gamma, d} V$ satisfies (SPL) by hypothesis and hence $\mathrm{PL}_{\text {loc }}(0)$ by Proposition 20 .

(ii) The restriction of $\pi$ to $T_{\gamma, d} V \cap\left(B\left(0, \varepsilon_{1}\right) \times\left(B^{n-2}\left(0,2 \varepsilon_{2}\right) \backslash\{0\}\right) \times \mathbb{C}\right)$ is unbranched.

(iii) If $\liminf \operatorname{in}_{t \rightarrow \infty} \omega(t) / t^{\delta}=0$ and if

$$
\left(z_{1}, \ldots, z_{n}\right) \in T_{\gamma, \delta} V \backslash\left(\mathbb{C} \times B^{n-2}\left(0, K_{2}\right) \times \mathbb{C}\right)
$$

satisfies $\left(z_{2}, \ldots, z_{n}\right) \in \mathbb{R}^{n-1}$, then also $z_{1}$ is real. Such a number $K_{2}$ exists by Proposition 20 .

(iv) The restriction of $\pi$ to $T_{\gamma, \delta} V \backslash\left(\mathbb{C} \times B^{n-2}\left(0, K_{2}\right) \times \mathbb{C}\right)$ is unbranched.

(v) If $w \in T_{\gamma, \delta} V$ satisfies $\left|\left(w_{2}, \ldots, w_{n-1}\right)\right| \leq 2 K_{2}$, then $\left|w_{1}\right| \leq K_{1}$.

For $d=1$, conditions (i) and (ii) are replaced by

$\left(\mathrm{i}^{\prime}\right)$ If $\left(z_{1}, \ldots, z_{n}\right) \in T_{\gamma, 1} V \cap\left(B\left(0, \varepsilon_{1}\right) \times B^{n-1}\left(0, \varepsilon_{2}\right)\right)$ satisfies $\left(z_{2}, \ldots, z_{n}\right)$ $\in \mathbb{R}^{n-1}$, then also $z_{1}$ is real.

$\left(i i^{\prime}\right)$ The restriction of $\pi$ to $T_{\gamma, 1} V \cap\left(B\left(0, \varepsilon_{1}\right) \times B^{n-1}\left(0,2 \varepsilon_{2}\right)\right) \backslash(\{(0, \ldots, 0)\}$ $\times \mathbb{C})$ is unbranched.

Now set, for $R>0$,

$$
\begin{aligned}
& \Gamma_{R}=\Gamma\left(\gamma, d, B\left(0, \varepsilon_{1}\right) \times B^{n-1}\left(0, \varepsilon_{2}\right), R\right), \\
& \Gamma_{R}^{\prime}=\Gamma\left(\gamma, \delta, B\left(0, K_{1}\right) \times B^{n-1}\left(0,2 K_{2}\right), R\right) .
\end{aligned}
$$

Fix a suitably large $R_{1}>0$ and define a semi-algebraic set by

$$
M=V \cap\left(\mathbb{C} \times \mathbb{R}^{n-1}\right) \cap \bar{\Gamma}_{R_{1}} \backslash \Gamma_{R_{1}}^{\prime}
$$

and a semi-algebraic function by

$h:\left[R_{1}, \infty\left[\rightarrow \mathbb{R}, \quad r \mapsto \sup \left\{|\operatorname{Im} x|:\right.\right.\right.$ there is $y \in \mathbb{R}^{n-2}$ with $\left.(x, y, r) \in M\right\}$.

By the curve selection lemma (see Hörmander [11, Theorem A.2.8]) there are $R_{2} \geq R_{1}$ and an algebraic curve $\sigma:\left[R_{2}, \infty[\rightarrow M\right.$ such that

$$
\pi_{n}(\sigma(r)) \equiv r \quad \text { and } \quad h(r) \equiv\left|\operatorname{Im} \pi_{1}(\sigma(r))\right| .
$$


We must have $\sigma \neq \gamma$ since $\gamma$ is real. Hence $\sigma$ admits a Puiseux series expansion

$$
\sigma(t)=\gamma(t)+\left(t^{\beta} w_{1}, t^{\beta} w^{\prime}, 0\right)+\widetilde{\sigma}(t)
$$

with $\beta \leq d, \mathbb{C} \times \mathbb{C}^{n-2} \ni\left(w_{1}, w^{\prime}\right) \neq 0,|\widetilde{\sigma}(t)|=o\left(t^{\beta}\right)$, and $\pi_{3} \circ \widetilde{\sigma} \equiv 0$. The definition of $M$ implies that $w^{\prime}$ and $\pi \circ \sigma$ are real. Since $h$ is semi-algebraic there are $C_{2}>0$ and $\alpha \leq \beta$ such that $h(t)=C_{2} t^{\alpha}+o\left(t^{\alpha}\right)$. Note that

$$
\liminf _{t \rightarrow \infty} \frac{\omega(t)}{t^{\alpha}}=0
$$

since $V$ is not $(\omega, \gamma, d, \delta)$-hyperbolic at 0 . Thus $T_{\zeta, \varrho} V$ satisfies (SPL) for each real simple curve $\zeta$ and each $\varrho \geq \alpha$ by hypothesis. The claim is now proved by looking at a complete set of cases and leading each one to a contradiction. When checking that all cases are covered, keep in mind that $\alpha \leq \beta \leq d$ and $\beta \geq \delta$; the latter holds since $\sigma(t) \notin \Gamma_{R_{1}}^{\prime}$ for all $t$.

(I) CASE $\beta=d<1$. Then $\left(w_{1}, w^{\prime}, 0\right) \in T_{\gamma, d} V$, and (i) implies that $w_{1}$ is real since $w^{\prime}$ is real. By construction, they do not both vanish, hence by (ii), $\left(w_{1}, w^{\prime}, 0\right)$ is a regular point of $T_{\gamma, d} V$. Note that $\alpha<\beta=d$ since $w_{1}$ is real. Thus 0 is a regular point of $T_{\operatorname{Re} \sigma, d} V$ which satisfies the hypotheses of Proposition 22 with $\alpha$ in place of $\delta$ and $\operatorname{Re} \sigma$ in place of $\gamma$. Proposition 22 implies that $z \in T_{\operatorname{Re} \sigma, \alpha} V$ is real whenever $\pi(z)$ is real. On the other hand, for $\zeta=i \lim _{t \rightarrow \infty} \operatorname{Im} \pi_{1}(\sigma(t)) t^{-\alpha} \neq 0$ we have $(\zeta, 0,0) \in T_{\operatorname{Re} \sigma, \alpha} V$. Since this is a contradiction, the present case is impossible.

$\left(\mathrm{I}^{\prime}\right)$ CASE $\beta=d=1$. This is the same as before except that (i) and (ii) are replaced by $\left(\mathrm{i}^{\prime}\right)$ and $\left(\mathrm{ii}^{\prime}\right)$, respectively.

(II) CASE $\delta<\beta<d$. In this case, the choice of $\delta$ implies that $\beta$ is not a critical value for $V$ and $\gamma$. Hence $T_{\gamma, \beta} V$ is homogeneous by Remark 11(d).

(A) Subcase $\alpha=\beta$. Since $T_{\gamma, \beta} V$ is homogeneous and since limit varieties are translation invariant by Remark $11(\mathrm{~b})$, the plane $E:=\mathbb{C} \cdot\left(w_{1}, w^{\prime}, 0\right)$ $+\mathbb{C} \cdot(0,0,1)$ is an irreducible component of $T_{\gamma, \beta} V$. Since $T_{\gamma, \beta} V=T_{0}\left(T_{\gamma, d} V\right)$ by Remark 11(d), inequality (2) holds for all $\left(z_{1}, \ldots, z_{n}\right) \in T_{\gamma, \beta} V$. Hence $w^{\prime} \neq 0$. On the other hand, $w_{1}$ is not real because we are in the case $\alpha=\beta$. Hence the real dimension of $E \cap \mathbb{R}^{n}$ is only 1, and Proposition 20 implies that $E$ does not satisfy (SPL). This is a contradiction since it is clear that (SPL) is inherited by irreducible components.

(B) Subcase $\alpha<\beta$. In this case, $w_{1}$ and $w^{\prime}$ are real. Since $T_{\gamma, \beta} V$ is two-dimensional, homogeneous, and invariant under all translations in the direction $(0, \ldots, 0,1)$, it is easy to see that all singular points are of the form $(0, \ldots, 0, \tau), \tau \in \mathbb{C}$. Hence $\left(w_{1}, w^{\prime}, 0\right)$ is a regular point of $T_{\gamma, \beta} V$ and the origin is a regular point of $T_{\operatorname{Re} \sigma, \beta} V$. Now Proposition 22 yields a contradiction as in case (I). 
(III) $\operatorname{CASE} \beta=\delta$.

(A) Subcase $\alpha=\beta$. Then

$$
\liminf _{t \rightarrow \infty} \omega(t) / t^{\delta}=\liminf _{t \rightarrow \infty} \omega(t) / t^{\alpha}=0 .
$$

Hence (iii) implies that $w_{1}$ is real since $w^{\prime}$ is real. This contradicts the hypothesis $\alpha=\beta$.

(B) Subcase $\alpha<\beta$. Then $\left|w^{\prime}\right|>2 K_{2}$ by (v) and the choice of $\sigma$. Hence it follows from (iv) that $T_{\gamma, \delta} V=T_{\gamma, \beta} V$ is regular at $\left(w_{1}, w^{\prime}, 0\right) \in \mathbb{R}^{n}$. Hence the origin is a regular point of $T_{\operatorname{Re} \sigma, \beta} V$. Again Proposition 22 yields a contradiction as in case (I).

As all possible cases lead to contradictions, we have finished the proof of the case where condition (c) of Definition 17 is violated. If condition (b) of Definition 17 is violated, then the above proof works with $\beta=-\infty$. In particular, case (III) cannot happen.

24. Theorem. Let $V$ be an algebraic surface in $\mathbb{C}^{3}$ and let $\omega$ be a weight function. Then $V$ satisfies $\mathrm{PL}(\omega)$ if and only if $T_{\gamma, d} V$ satisfies (SPL) for all real simple curves $\gamma$ and all $d \in] 0,1]$ with $\liminf _{t \rightarrow \infty} \omega(t) / t^{d}=0$.

Proof. The result follows immediately from Proposition 23 together with Theorem 18.

25. REMARK. It should be pointed out that for working examples the approach in [5], which is more algorithmic in spirit, is often very useful. Also, a direct proof of Theorem 24, i.e., a proof without recourse to hyperbolicity conditions, would facilitate the generalization of the result to higher dimensions. Unfortunately, no such proof is known yet.

26. Corollary. For $P \in \mathbb{C}\left[z_{1}, z_{2}, z_{3}\right]$ with $\operatorname{deg} P \geq 1$ assume that $V(P)_{h}$ satisfies (SPL). Let $M$ consist of all those $\left.\left.d \in\right] 0,1\right]$ such that $T_{\gamma, d} V(P)$ does not satisfy (SPL) for some real simple curve $\gamma$. Set $c:=$ $\sup M$ if $M \neq \emptyset$, and set $c:=0$ otherwise. Then for any weight function $\omega$ the variety $V(P)$ satisfies $\mathrm{PL}(\omega)$ if and only if $t^{c}=O(\omega(t))$ for $t \rightarrow \infty$. Moreover, the number $c$ is rational.

Proof. All of this follows immediately from Theorem 24 with the exception of the fact that $c$ is rational, which follows from [4, Theorem 22].

Before we can prove the analogue to Theorem 24 for (SPL) we have to recall Definitions 3.12 and 3.15 from [7].

27. Definition.

(a) Let $d \leq 1$. Two real simple curves $\gamma$ and $\sigma$ are equivalent modulo $d$ if for each $R>0$ and each neighborhood $U$ of zero we have

$$
\Gamma(\gamma, d, U, R) \cap \Gamma(\sigma, d, U, R) \neq \emptyset .
$$


(b) Let $V \subset \mathbb{C}^{n}$ be an algebraic variety, let $\gamma$ be a real simple curve, and let $d \leq 1$. A real, singular point $\zeta$ of $T_{\gamma, d} V$ is called terminating for $\gamma$ and $d$ if there is a real simple curve $\sigma$ which is equivalent to $\gamma$ modulo $d$ such that $\zeta$ is a simple singularity of $T_{\sigma, d} V$ in the sense of Definition 14, i.e., there is no critical value for $V$ and $\sigma(t)+t^{d} \zeta$ that is strictly smaller than $d$.

28. Definition. Let $V$ be an algebraic variety in $\mathbb{C}^{n}$. We say that $V$ is hyperbolic in conoids if for each real simple curve $\gamma$ and each $d \leq 1$ the following conditions are satisfied:

(1) $T_{\gamma, d} V$ satisfies (SPL).

(2) If $\xi \in T_{\gamma, d} V \cap \mathbb{R}^{n}$ is either a regular point of $T_{\gamma, d} V$ or a singularity of $T_{\gamma, d} V$ which is terminating for $\gamma$ and $d$, then $V$ is $(0, \gamma, d)$-hyperbolic at $\xi$.

(3) If $\xi \in T_{\gamma, d} V \cap \mathbb{R}^{n}$ is a singularity of $T_{\gamma, d} V$ which is not terminating for $\gamma$ and $d$, then $V$ is $(0, \gamma, d, \delta)$-hyperbolic at $\xi$ for $\delta:=\Delta(\gamma, d, \xi)$.

29. TheOrem. Let $V$ be an algebraic surface in $\mathbb{C}^{n}$ that satisfies $\mathrm{PL}_{\text {loc }}(\xi)$ at each $\xi \in V \cap \mathbb{R}^{n}$. The following are equivalent:

(a) $V$ satisfies (SPL).

(b) $T_{\gamma, d} V$ satisfies (SPL) for all real simple curves $\gamma$ and all $d \leq 1$.

(c) $V$ is 0-hyperbolic in conoids in the sense of Definition 17.

(d) $V$ is hyperbolic in conoids in the sense of Definition 28.

Proof. The equivalence of (a) and (d) is shown in [7, Theorem 4.4], the equivalence of (b) and (c) is shown in Proposition 23, while (d) obviously implies (b).

We complete the proof by showing that (c) implies (d). To do so, fix $\xi \in T_{\gamma, d} V \cap \mathbb{R}^{n}$. If $\xi$ is regular, the two notions of hyperbolicity coincide. If $\xi$ is singular but not terminating, then $\xi$ is not simple, and (c) implies that $V$ is $(0, \gamma, d, \delta)$-hyperbolic at $\xi$ for $\delta:=\Delta(\gamma, d, \xi)$. If $\xi$ is singular and terminating, then there is a real simple curve $\sigma$, equivalent to $\gamma$ modulo $d$, such that $\xi$ is a simple singularity of $T_{\sigma, d} V$. Then (c) implies that $V$ is $(0, \sigma, d)$-hyperbolic at $\xi$. It is easy to see that this implies that $V$ is $(0, \gamma, d)$-hyperbolic at $\xi$.

\section{References}

[1] R. W. Braun, Hörmander's Phragmén-Lindelöf principle and irreducible singularities of codimension 1, Boll. Un. Mat. Ital. A (7) 6 (1992), 339-348.

[2] R. W. Braun, R. Meise, and B. A. Taylor, Ultradifferentiable functions and Fourier analysis, Result. Math. 17 (1990), 207-237.

[3] - - - - - The geometry of analytic varieties satisfying the local Phragmén-Lindelöf condition and a geometric characterization of the partial differential operators that are surjective on $\mathcal{A}\left(\mathbb{R}^{4}\right)$, Trans. Amer. Math. Soc. 356 (2004), 1315-1383. 
[4] R. W. Braun, R. Meise, and B. A. Taylor, Optimal Gevrey classes for the existence of solution operators for linear partial differential operators in three variables, J. Math. Anal. Appl. 297 (2004), 852-868.

[5] - - - - Characterization of the linear partial differential equations that admit solution operators on Gevrey classes, J. Reine Angew. Math. 588 (2005), 169-220.

[6] - , - - , Higher order approximations at infinity to algebraic varieties, Note Mat., to appear.

[7] - - - - , The algebraic surfaces on which the classical Phragmén-Lindelöf theorem holds, Math. Z., to appear.

[8] -, - - - Higher order tangents to analytic varieties along curves II, Canad. J. Math., to appear.

[9] E. M. Chirka, Complex Analytic Sets, Kluwer, Dordrecht, 1989; translated from: Nauka, Moscow, 1985.

[10] L. Hörmander, On the existence of real analytic solutions of partial differential equations with constant coefficients, Invent. Math. 21 (1973), 151-182.

[11] —, The Analysis of Linear Partial Differential Operators II, Springer, Berlin, 1983.

[12] R. Meise and B. A. Taylor, Phragmén-Lindelöf conditions for graph varieties, Result. Math. 36 (1999), 121-148.

[13] R. Meise, B. A. Taylor, and D. Vogt, Characterization of the linear partial differential operators with constant coefficients that admit a continuous linear right inverse, Ann. Inst. Fourier (Grenoble) 40 (1990), 619-655.

[14] - , - - Continuous linear right inverses for partial differential operators on nonquasianalytic classes and on ultradistributions, Math. Nachr. 180 (1996), 213-242.

Mathematisches Institut

Heinrich-Heine-Universität

Universitätsstr. 1

40225 Düsseldorf, Germany

E-mail: Ruediger.Braun@uni-duesseldorf.de meise@math.uni-duesseldorf.de
Department of Mathematics University of Michigan Ann Arbor, MI 48109, U.S.A. E-mail: taylor@umich.edu 